\title{
Triphora uniflora A. C. Ferreira, Baptista \& Pansarin (Orchidaceae: Triphoreae): uma nova espécie e primeiro registro do gênero Triphora Nutt. para o estado de São Paulo, Brasil
}

\author{
Alessandro Wagner Coelho Ferreira ${ }^{1,4}$, Dalton Holland Baptista ${ }^{2}$ e Emerson Ricardo Pansarin ${ }^{3}$
}

Recebido em 17/04/2009. Aceito em 24/11/2009

RESUMO - (Triphora uniflora A.C. Ferreira, Baptista \& Pansarin (Orchidaceae: Triphoreae): uma nova espécie e primeiro registro do gênero Triphora Nutt. para o estado de São Paulo, Brasil). Triphora uniflora A. C. Ferreira, Baptista \& Pansarin uma nova espécie de Orchidaceae, é descrita e ilustrada. Além disso, o gênero Triphora é referido pela primeira vez para o estado de São Paulo. As relações da nova espécie com outros táxons do gênero, bem como a necessidade de conservação do habitat natural dessa espécie de Triphora, são discutidas.

Palavras-chave: Brasil, nova espécie, Orchidaceae, Triphoreae, Triphorinae

ABSTRACT - (Triphora uniflora A.C. Ferreira, Baptista \& Pansarin (Orchidaceae: Triphoreae): a new species and the first record of the genus Triphora Nutt. for São Paulo state, Brazil). Triphora uniflora A. C. Ferreira, Baptista \& Pansarin, a new species of Orchidaceae, is described and illustrated. Furthermore, this is the first report of the genus Triphora for São Paulo state, Brazil. The relationship of this new species to other taxa of the genus and the need to preserve the natural habitat of this Triphora species are discussed.

Key words: Brazil, new species, Orchidaceae, Triphoreae, Triphorinae

\section{Introdução}

O gênero Triphora Nutt. (Orchidaceae: Triphoreae: Triphoriinae) é constituído por 19 espécies terrestres distribuídas desde o Sul do Canadá, passando pelas Índias Ocidentais, até a América do Sul (Rothacker 2005), onde ocorre até o norte da Argentina (Johnson 2001). No Brasil, o gênero está representado por cerca de cinco espécies distribuídas pelos estados do Amazonas, Pará, Goiás, Minas Gerais e Distrito Federal (Hoehne 1940; Pabst \& Dungs 1975; Batista \& Bianchetti 2003; Pridgeon et al. 2003).

O trabalho tem como objetivo apresentar a descrição de um novo táxon pertencente ao gênero Triphora Nutt. Essa espécie foi encontrada durante a realização do inventário florístico das Orchidaceae ocorrentes na região central do estado de São Paulo. O gênero é referido pela primeira vez para o estado de São Paulo.

\section{Material e métodos}

O estudo foi realizado na região central do estado de São Paulo, no município de São Carlos, em duas áreas: na reserva legal da Fazenda Canchim (21⒌' $12^{\prime \prime} \mathrm{Se}$ e $\left.7^{\circ} 50^{\prime} 17^{\prime \prime} \mathrm{W}\right)$, com cerca de 112 ha e, num fragmento com cer-

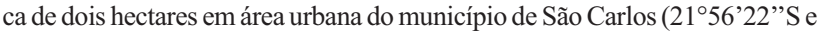
4749'19”'W, 780 m). A porção central do estado é caracterizada por ser uma região ecotonal entre os biomas do Cerrado e da Floresta Atlântica (Kronka et al. 1993; Soares et al. 2003). No entanto, como conseqüência da intensa atividade econômica, principalmente a partir dos anos de 1960 a vegetação nativa foi reduzida a pequenos fragmentos (Kronka et al. 1998; Soares et al. 2003). A altitude média da região é de 850 metros. O clima predominante, segundo a classificação de Köppen (1948), é o Cwa (subtropical úmido), com verões quentes e chuvosos e invernos frios e secos.
Os indivíduos da espécie aqui descrita foram coletados em fevereiro de 2006 (autorização IBAMA, processo número 02001.003951/200650 e Instituto Florestal de São Paulo: processo número 40.380/2006), e mantidos em cultivo em casa de vegetação até a ocorrência da floração. A descrição da espécie e as ilustrações foram feitas a partir de material vivo. As flores e os detalhes das peças florais foram ilustrados como auxílio de um estereomicroscópio acoplado à câmara clara. A terminologia usada na descrição das estruturas vegetativas e florais seguiu Harris \& Harris (1994). As características específicas de Orchidaceae foram baseadas em Dressler (1993). Exemplares da espécie foram herborizados e estão depositados no herbário da Universidade Estadual de Campinas (UEC). Espécimes depositados nos herbários CEN e SPFR também foram analisados.

\section{Resultados e discussão}

Triphora uniflora A.C. Ferreira, Baptista \& Pansarin sp. nov. Fig. 1 A-G.

Triphora uniflora ab omnibus aliis speciebus sui generis caule brevi, sitis gracili, unifloro, foliis cordatis vel ovatis, labello trilobato cum lobo intermedio rotundo cum marginibus undulatis et apice recurvo distinguenda; a T. pusilla affinis, sed folliis maioribus, patulis, floribus subalbidus, labello cum lobis lateralibus falcatis, lobo intermedio vinaceo, albo-venulato, carina centrali cum lamellis flavescentibus vel subviridis, columna longa et clavata differt.

Erva terrestre; raízes tuberosas $1,5-3,0 \times 0,6-1,1 \mathrm{~cm}$, cilíndricas, com as extremidades arredondadas, paucipilosas, de coloração creme-esbranquiçadas, interligadas por porções de rizoma. Rizoma ca. 1,5-3,0 cm. Caule aéreo 6-11 x 0,15-0,18 cm, cilíndrico, ereto, violáceo principalmente nos $2 / 3$ basais, com 3-5 folhas. Folhas $0,8-1,4 \times 0,7-1,2 \mathrm{~cm}$, glabras, membranáceas, com margens inteiras; as basais

\footnotetext{
1 Programa de Pós-Graduação em Ecologia e Recursos Naturais da Universidade Federal de São Carlos, Departamento de Botânica, São Carlos, SP, Brasil 2 Projeto Orchidstudium, Piracicaba, São Paulo, Brasil

3 Universidade de São Paulo, Faculdade de Filosofia, Ciências e Letras de Ribeirão Preto, Departamento de Biologia, Ribeirão Preto, SP, Brasil

4 Autor para correspondência: alessandrowcf@yahoo.com.br
} 


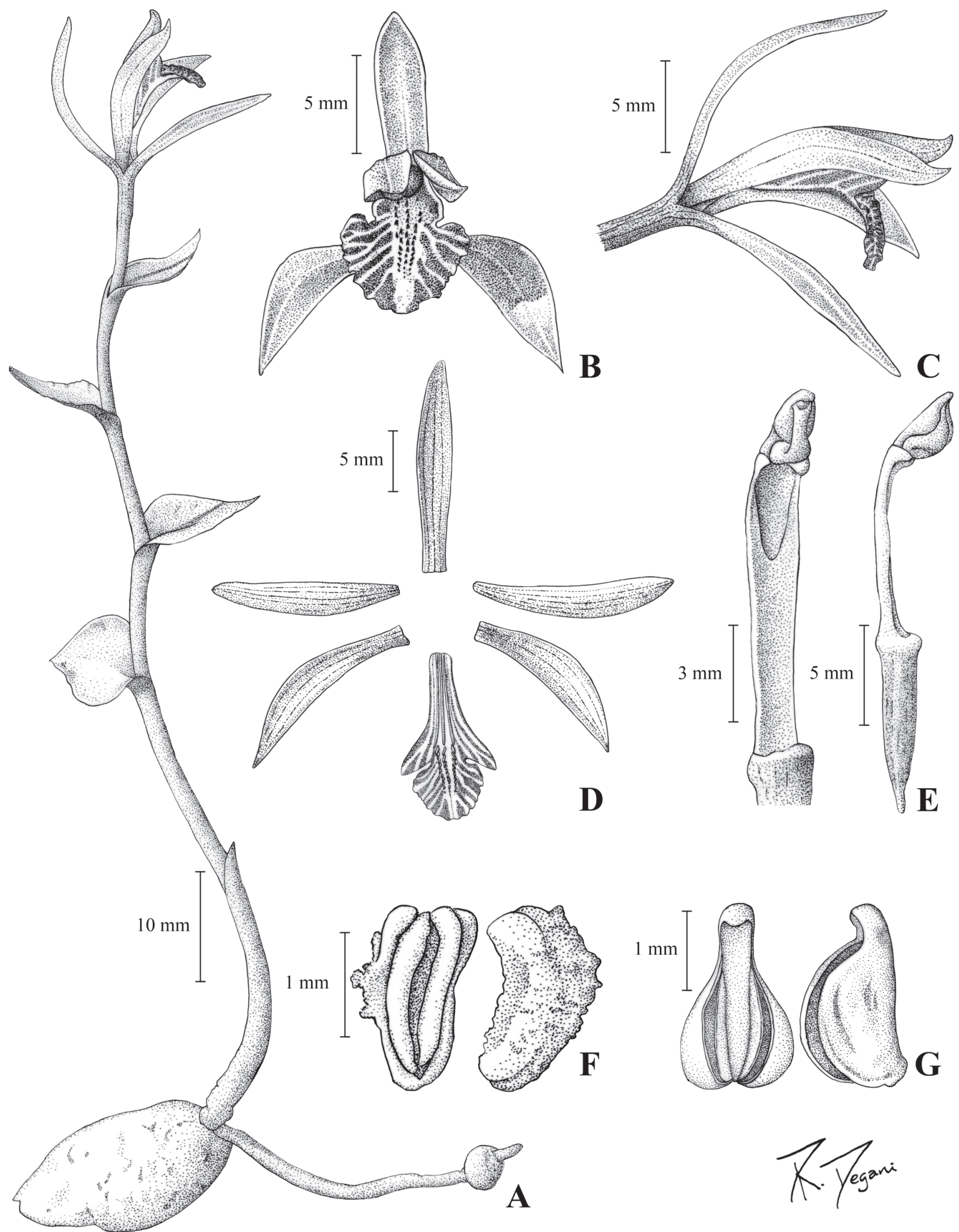

Figura 1. Triphora uniflora A.C. Ferreira, Baptista \& Pansarin (A.W.C. Ferreira 148370). A. Hábito com flor. B. Flor em vista frontal. C. Flor em vista lateral. D. Peças florais. E. Coluna em vista abaxial (esquerda); coluna e ovário em vista lateral (direita). F. Polinário em vistas: abaxial (esquerda); lateral-adaxial (direita). G. Antera em vistas: abaxial (esquerda) e lateral (direita). 
cordadas, verde-escuras na porção adaxial e arroxeadas na face abaxial, com bordas levemente crenadas; as apicais ovais, verdes na porção adaxial e verdes com faixas violáceas longitudinais na região das nervuras na face abaxial. Inflorescência racemosa, apical, portando uma única flor. Flor de coloração predominantemente esbranquiçada a rosa claro, glabras, ressupinadas; sépala dorsal 1,3-1,7 x 0,2-0,25 cm, elíptico-lanceolada, envolvendo levemente a coluna; sépalas laterais $1,2-1,4 \times 0,3 \mathrm{~cm}$, elíptico-lanceoladas, levemente encurvadas para baixo; pétalas 1,5-1,7 x 0,15-0,3 cm, oblanceoladas, falcadas, branco-hialinas, projetadas para frente, ápice agudo, margem inteira; labelo 1,3-1,7 x 0,15-0,20 cm, trilobado, porção mediana com três carenas longitudinais e paralelas do meio para o ápice; carenas amarelo-esverdeadas, compostas por papilas irregulares; lobos laterais $0,3-0,4 \mathrm{x}$ 0,15-0,20 cm, oblongos, projetados para frente; lobo apical arredondado, púrpura com estrias oblíquas e esbranquiçadas, ápice recurvado, margem ondulada. Coluna 1,0-1,2 x 0,10-0,25 cm, oblanceolada, alongada, delgada, esverdeada nos $2 / 3$ basais, esbranquiçadas em direção ao ápice; estigma longitudinalmente oval, alongado, levemente côncavo, com superfície lisa, esbranquiçado; ovário + pedicelo 1,6-2,2 cm x 0,15-0,20 cm ínfero, clavado, alongado, glabro, levemente púrpura na base, esverdeado em direção ao ápice; antera 2-4 x 1-1,5 cm, ungüiculada, persistente, púrpura, face adaxial arredondada, face abaxial e com dois septos onde se encaixam as políneas; polinário com quatro políneas púrpuras de aspecto farináceo; viscídio rudimentar.

Tipo: Brasil. São Paulo: São Carlos, Fazenda Canchim, 20/II/2007 (fl.), A.W.C. Ferreira 148370 (Holótipo UEC).

Parátipos: Brasil. São Paulo: São Carlos, Jardim Acapulco, 12/III/2009 (fl.), E.R. Pansarin \& A.W.C. Ferreira 1286 (SPFR). Distrito Federal: Brasília, Jardim Botânico de Brasília, Mata do Córrego Cabeça de Veado. 12/III/2003 (fl.), J.A.N. Batista \& K.F. Pellizaro 1401 (CEN, SPFR).

Triphora uniflora A.C. Ferreira, Baptista \& Pansarin pode ser distinguida de Triphora pusilla (Rchb.f.) Schltr., espécie mais proximamente relacionada ao táxon aqui descrito, por apresentar folhas evidentes e patentes, lobos laterais do labelo falcados, flores predominantemente esbranquiçadas, labelo vináceo com estrias brancas, carenas centrais amareladas a esverdeadas e coluna delgada e clavada. As flores de Triphora uniflora também lembram muito as de T. trianthophora (Sw.) Rydb. No entanto, T. uniflora é facilmente distinguida de $T$. trianthophora pela presença do labelo vináceo com estrias oblíquas brancas, por emitir apenas uma flor, pela superfície abaxial da folha arroxeada e por apresentar caule de tamanho consideravelmente menor. A espécie T. uniflora parece se tratar do mesmo táxon encontrado no Parque Nacional do Iguaçu, localizado ao norte da Argentina, e determinado apenas ao nível de gênero, Triphora sp. (Johnson 2001). Assim, de acordo com os dados aqui apresentados, T. uniflora possui ampla distribuição, ocorrendo desde o centro-oeste do Brasil, até a porção norte da Argentina, e o gênero Triphora é referido pela primeira vez para o estado de São Paulo (Hoehne 1940; Pabst \& Dungs 1975; Pridgeon et al. 2003).

Etimologia: o epíteto específico (uniflora) refere-se à produção de uma única flor na época da floração. Todos os indivíduos avistados nas populações analisadas produziram apenas um único botão floral apical.

Durante o período de coleta pela região central do estado de São Paulo foram identificadas apenas duas pequenas populações em fragmentos de florestas estacionais semideciduais. Uma delas foi encontrada na Fazenda Canchim ( $21^{\circ} 57^{\prime} 12^{\prime \prime} \mathrm{S}$ e $\left.47^{\circ} 50^{\prime} 17^{\prime \prime} \mathrm{W}, 850 \mathrm{~m}\right)$ e a outra em uma região de aproximadamente dois hectares em área urbana do município de São Carlos (2156'22"S e 4749'19”W, 780 m).

No início do mês de janeiro, provavelmente sob influência da elevada precipitação que ocorre na região durante o verão, cada planta emite um único caule aéreo não ramificado. Um único botão floral é emitido logo em seguida, que se desenvolve voltado para baixo, paralelamente ao caule, adotando a posição ereta apenas nos momentos que antecedem a antese. Cada flor dura menos de 24 horas. As plantas são gregárias com picos de floração nos quais muitas plantas da população florescem simultaneamente. Durante um período de floração normalmente ocorrem até quatro ou cinco picos de floração. Isso possibilita que nem todas as plantas floresçam ao mesmo tempo. O período de floração de Triphora uniflora se estende desde o final de fevereiro até o início do mês de março. A tendência de muitas flores abrirem ao mesmo tempo pode estar relacionada à otimização da ação dos polinizadores, um fenômeno que tem sido documentado para outras Triphoreae, como Triphora trianthophora (Williams 1994) e Psilochilus modestus Barb. Rodr. (Pansarin \& Amaral 2008).

Apesar de recém descoberta, Triphora uniflora pode ser enquadrada no status de EN (em perigo), de acordo com os critérios e categorias estabelecidas pela IUCN (2001). Triphora uniflora é uma espécie altamente ameaçada de extinção no estado de São Paulo devido às suas reduzidas populações nos locais de estudo. As áreas de florestas estacionais semideciduais do interior desse estado foram praticamente dizimadas nas últimas décadas, inicialmente para o plantio de café e, mais recentemente devido à expansão das lavouras de cana-de-açúcar e, de acordo com Viana \& Pinheiro (1998), grande parte da diversidade encontra-se distribuída entre esses pequenos fragmentos de áreas nativas. Assim, como apontado previamente por Myers et al. (2000), o enquadramento de fragmentos de Floresta Atlântica sensu lato como áreas de preservação ("hotspots"), torna-se prioritário, uma vez que o bioma da Mata Atlântica conta atualmente com apenas 5-8\% de sua formação original (Dean 1995).

\section{Agradecimentos}

À Profa. Dra. Maria Inês Salgueiro Lima (orientadora do primeiro autor), ao tecnólogo Carlos Aparecido Casali (apoio em campo), ambos do Departamento de Botânica da Universidade Federal de São Carlos (UFSCar) e aos funcionários do Programa de Pós-Graduação em Ecologia 
e Recursos Naturais da UFSCar pelo apoio nos trabalhos em campo; ao ilustrador botânico Ricardo Milanetti Degani pelas imagens; a Américo Docha Neto do projeto Orchidstudium pela ajuda na pesquisa; a Tarcísio de Sousa Filgueiras pela revisão da diagnose latina; ao IBAMA, Instituto Florestal de São Paulo e direção da Fazenda Canchim, que permitiram o acesso aos fragmentos florestais; ao contribuinte brasileiro e a CAPES pela bolsa de doutoramento concedida ao primeiro autor.

\section{Referências bibliográficas}

Batista, J.A.N. \& Bianchetti, L.B. 2003. Lista atualizada das Orchidaceae do Distrito Federal. Acta Botanica Brasilica 17: 183-201.

Dean, W. 1995. A ferro e fogo: a história e a devastação da Mata Atlântica brasileira. São Paulo, Companhia das Letras.

Dressler R.L. 1993. Phylogeny and classification of the orchid family. Cambridge, Cambridge University Press.

Harris, J.G. \& Harris, M.W. 1994. Plant identification terminology: an illustrated glossary. Utah, Spring Lake Publishing.

Hoehne, F.C. 1940. Orchidaceas. Pp. 241-244. In: F. C. Hoehne (ed.). Flora Brasilica XII (I). São Paulo, Secretaria de Agricultura, Indústria e Comércio de São Paulo.

IUCN. 2001. IUCN Red List Categories and Criteria, Version 3.1. Prepared by the IUCN Species Survival Commission. Cambridge, IUCN.

Johnson, A.E. 2001. Las orquídeas del Parque Nacional Iguazú. Pp. 210-211. Buenos Aires, Editora L.O.L.A.

Köppen, W. 1948. Climatologia: con un estudio de los climas de la tierra. MéxicoD.F., Fondo de Cultura Econômica.
Kronka, F.J.M.; Matsukuma, C.K.; Nalon, M.A.; Cali, I.H.D.; Rossi, M.; Mattos, I.F.A.; Shin-Ike, M.S. \& Pontinhas, A.A.S. 1993. Inventário florestal do Estado de São Paulo. São Paulo, Instituto Florestal de São Paulo.

Kronka, F.J.N.; Nalon, M.A.; Matsukuma, C.K.; Pavão, M.; Guillaumon, J.R.; Cavalli, A.C.; Giannotti, E.; Iwane, M.S.S.; Lima, L.M.P.R.; Montes, J.; Del Cali, I.H. \& Haack, P.G. 1998. Áreas de Domínio do Cerrado no Estado de São Paulo. São Paulo, Instituto Florestal.

Myers N.; Mittermeier, R.A.; Mittermeier, C.G.; Fonseca, G.A.B. \& Kent, J. 2000. Biodiversity hotspots for conservation priorities. Nature 403: 853-858.

Pabst, G.F.J. \& Dungs, F. 1975. Orchidaceae Brasiliensis, v.I. Hildesheim, Brücke-Verlag Kurt Schmersow.

Pansarin, E.R. \& Amaral, M.C.E. 2008. Pollen and nectar as a reward in the basal epidendroid Psilochilus modestus (Orchidaceae: Triphoreae): A study of floral morphology, reproductive biology and pollination strategy. Flora 203: 474-483.

Pridgeon, A.M.; Cribb, P.J.; Chase, M.W. \& Rasmussen, F.N. 2003. Genera Orchidacearum vol. 3, Orchidoideae (Part 2), Vanilloideae. London, Oxford University Press.

Rothacker, E.P. 2005. Tribe Triphoreae. Pp. 607-618. In: Pridgeon, A.M.; Cribb, P.J.; Chase, M.W. \& Rasmussen, F.N. (eds.), Genera Orchidacearum vol. 4. New York, Oxford University Press.

Soares, J.J.; Silva, D.W. \& Lima, M.I.S. 2003. Current state and projection of the probable original vegetation of the São Carlos region of São Paulo State, Brazil. Brazilian Journal of Biology 63: 527-536.

Viana, V.M. \& Pinheiro, L.A.F.V. 1998. Conservação da biodiversidade em fragmentos florestais. Série Técnica IPEF 12: 25-42.

Williams, S.A. 1994. Observations on reproduction in Triphora trianthophora (Orchidaceae). Rhodora 96: 30-43. 